\section{BRAZIULIAN JOURNAL \\ OF MEDICAL AND BIOLOGICAL RESEARCH}

www.bjournal.com.br
ISSN 1414-431X

Volume 45 (12) 1102-1340 December 2012

\section{BIOMIDICAL SCIENCES}

AND

CLINICAL INVESTIGATION

Braz J Med Biol Res, December 2012, Volume 45(12) 1209-1214

doi: 10.1590/S0100-879X2012007500133

High-frequency electroacupuncture versus carprofen in an incisional pain model in rats

F.M. Teixeira, L.L. Castro, R.T. Ferreira, P.A. Pires, F.A. Vanderlinde and M.A. Medeiros

The Brazilian Journal of Medical and Biological Research is partially financed by

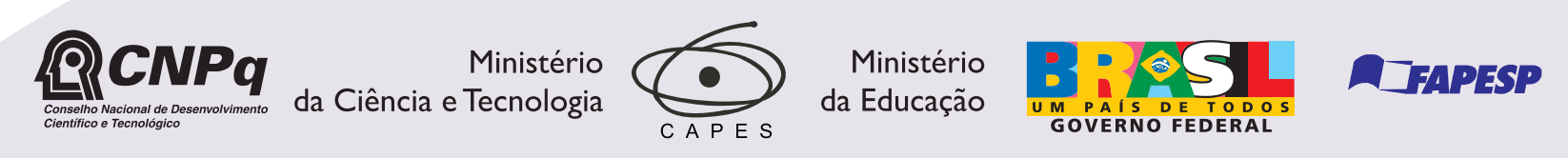

Scie/
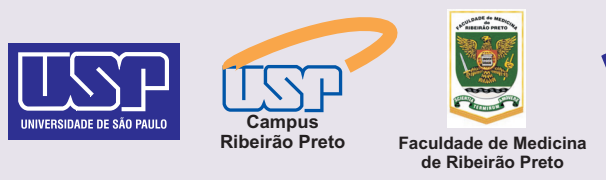

Institutional Sponsors

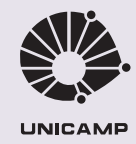

SHIMADZU

$\oplus$ UNICAMP

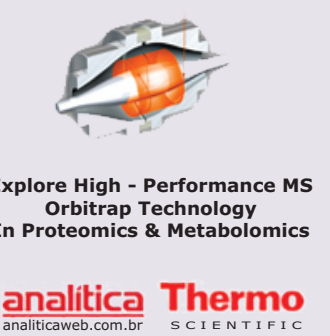




\title{
High-frequency electroacupuncture versus carprofen in an incisional pain model in rats
}

\author{
F.M. Teixeira*, L.L. Castro*, R.T. Ferreira, P.A. Pires, F.A. Vanderlinde \\ and M.A. Medeiros \\ Departamento de Ciências Fisiológicas, \\ Universidade Federal Rural do Rio de Janeiro, Rio de Janeiro, RJ, Brasil
}

\begin{abstract}
The objective of the present study was to compare the effect of electroacupuncture (EA) and carprofen (CP) on postoperative incisional pain using the plantar incision $(\mathrm{PI})$ model in rats. A 1-cm longitudinal incision was made through skin, fascia and muscles of a hind paw of male Wistar rats and the development of mechanical and thermal hypersensitivity was determined over 4 days using the von Frey and Hargreaves methods, respectively. Based on the experimental treatments received on the third postoperative day, the animals were divided into the following groups: $\mathrm{PI}+\mathrm{CP}(\mathrm{CP}, 2 \mathrm{mg} / \mathrm{kg}, \mathrm{po})$; PI+EAST36 (100-Hz EA applied bilaterally at the Zusanli point (ST36)); PI+EANP (EA applied to a non-acupoint region); PI+IMMO (immobilization only); PI (vehicle). In the von Frey test, the PI+EAST36 group had higher withdrawal force thresholds in response to mechanical stimuli than the $\mathrm{PI}, \mathrm{PI}+\mathrm{IMMO}$ and $\mathrm{PI}+\mathrm{EANP}$ groups at several times studied. Furthermore, the PI+EAST36 group showed paw withdrawal thresholds in response to mechanical stimuli that were similar to those of the PI+CP group. In the Hargreaves test, all groups had latencies higher than those observed with PI. The PI+EAST36 group was similar to the PI+IMMO, PI+EANP and $\mathrm{PI}+\mathrm{CP}$ groups. We conclude that $100-\mathrm{Hz}$ EA at the ST36 point, but not at non-acupoints, can reduce mechanical nociception in the rat model of incisional pain, and its effectiveness is comparable to that of carprofen.
\end{abstract}

Key words: Hyperalgesia; Plantar incision; Acupuncture; Zusanli point; Non-steroidal anti-inflammatory

\section{Introduction}

Postoperative incisional pain is simultaneously a unique and common form of acute pain. Appropriate treatment reduces morbidity following surgery, improves outcomes and reduces clinical costs (1). However, despite the development of new drugs and improved analgesic techniques, postoperative incisional pain remains under-evaluated and poorly treated, as demonstrated by a survey showing that about 50 to $70 \%$ of patients experience moderate to severe pain after surgery (2). Different pathophysiological mechanisms are responsible for the pain caused by inflammation, nerve injury or incision. This explains why many treatment strategies are effective only against specific types of pain (3).

An animal model used to study postoperative pain is a 1-cm incision through the skin and muscle of the plantar surface of the rat hind paw (4). This experimental model is characterized by spontaneous pain, mechanical allodynia and thermal hyperalgesia that lasts for several days, and which corresponds with the time course of postoperative incisional pain in patients (5). Several classes of drugs are effective in rat models of incisional pain, including gabapentin, morphine (5) and the non-steroidal anti-inflammatory drugs (NSAIDs) of both the cyclooxygenase (COX)-2 selective (5) and COX-1/COX-2 non-selective (including indomethacin) varieties (5). Carprofen (CP) is an NSAID, a propionic acid derivative, that preferentially inhibits COX-2, producing anti-inflammatory, analgesic and antipyretic activities. The efficacy of $\mathrm{CP}$ in controlling postoperative incisional pain has been demonstrated in several species including rodents (6). However, despite its preferential COX-2 inhibition, CP has the potential to cause damage to the gastrointestinal tract, kidneys and liver (7). Electroacupuncture (EA) has been proposed as a low-cost and practical technique that achieves effective pain control with minimal side effects (8). The effect of acupuncture on postoperative pain has been studied clinically and experimentally. Although clini-

Correspondence: M.A. Medeiros, Departamento de Ciências Fisiológicas, UFRRJ, BR 465, km 7, 23890-000 Seropédica, RJ, Brasil. Fax: +55-21-2682-3222. E-mail: magda.medeiros@gmail.com

*These authors contributed equally to this study.

Received March 26, 2012. Accepted August 9, 2012. Available online August 24, 2012. Published December 17, 2012. 
cal results have been contradictory (9), experimental data clearly demonstrate that EA can produce potent analgesic effects in models of acute or chronic inflammatory pain $(10,11)$. Oliveira and Prado (11) have shown that a 15-min period of low-frequency EA applied 120 min after surgery to the Zusanli (ST36) and Sanyinjiao (SP6) points, but not to non-acupoints, can reduce mechanical hyperalgesia in the plantar incisional (PI) pain model. In the present study, we assessed the efficacy of high-frequency EA applied on the third day after surgery in reducing mechanical and thermal hyperalgesia induced by $\mathrm{PI}$ in rats, and compared it to the anti-inflammatory drug $\mathrm{CP}$, commonly prescribed to relieve postoperative pain.

\section{Material and Methods}

Experiments were carried out on adult male Wistar rats (300-350 g) obtained from the Departamento de Ciências Fisiológicas, Universidade Federal Rural do Rio de Janeiro. The experiment was approved by the Ethics Committee on the Use of Human and Animal Subjects in Teaching and Research of Universidade Federal Rural do Rio de Janeiro, and all efforts were made to ensure that both animal numbers and suffering were minimized in accordance with the guidelines for experimental pain in conscious animals recommended by the International Association for the Study of Pain (12). Rats were housed in groups of 4 animals per cage with a 12-h light/dark cycle and food and water were available ad libitum.

PI was performed as previously described (4). Briefly, under anesthesia with ketamine $(50 \mathrm{mg} / \mathrm{kg}, i p)$ and xylazine (25 mg/kg, ip), the plantar surface of the right hind paw was prepared with a povidone-iodine solution. A midline longitudinal incision $(1 \mathrm{~cm}$ ) was made starting $0.5 \mathrm{~cm}$ from the heel. The plantar fascia and muscle were elevated and incised longitudinally. The wound was closed with 5-0 nylon sutures. For sham procedures, animals were anesthetized with ketamine and xylazine, and the sole of the right hind paw was prepared in a sterile manner with povidone-iodine solution. A systemic antibiotic (30,000 IU, im, Pentabiotic, Fort Dodge, Brazil) consisting of benzathine penicillin, procaine benzyl penicillin, benzyl penicillin potassium, dihydrostreptomycin sulfate, and streptomycin sulfate was administered to all rats.

To determine the effects of EA and CP on the time course of hyperalgesia, treatments were administered on the third postoperative day and behavioral tests were repeated at 30 min and at 1, 3, 6, 9, 12, and $24 \mathrm{~h}$ after the treatments.

In the EA treatment groups, EA was bilaterally applied at the ST36 (PI+EAST36, $\mathrm{N}=8)$ point or at a non-acupoint site (PI+EANP, $N=8)$. Stainless steel needles $0.3 \mathrm{~mm}$ in diameter were inserted $5 \mathrm{~mm}$ deep into ST36 (Zusanli), which is located between the tibia and the fibula approximately $5 \mathrm{~mm}$ lateral to and below the anterior tubercle of the tibia (13). The non-acupoint site was located $5 \mathrm{~mm}$ lateral to the midline of the posterior surface of the hind limb. Each needle was independently stimulated for 20 min with an electrical current of faradic, bipolar and asymmetrical waves at $100 \mathrm{~Hz}$ using an asymmetric F1000 apparatus (Lautz, Brazil). Stimulus intensity was set at twice the threshold for a detectable muscle twitch (between 2 and $4 \mathrm{~V}$ ). To perform $E A$, the animals were immobilized in a plastic cylinder (14) and the PI+IMMO group ( $\mathrm{N}=7$ ) was used as control for the effects of immobilization. In this group, the animals were immobilized in the same apparatus for the same period of time, but with no needle insertion or electrical stimulation.

Carprofen (Rymadil ${ }^{\circledR}$, Pfizer Laboratory, Brazil) was used at the dose of $2 \mathrm{mg} / \mathrm{kg}$ (15), diluted in water to a concentration that allowed the administration of a constant volume of $10 \mathrm{~mL} / \mathrm{kg}$, and administered by gastric gavage. In the PI group $(\mathrm{N}=6)$, rats were subjected to plantar incision and water gavage (PI), and in the sham-incision group ( $\mathrm{NPI}, \mathrm{N}=6$ ) the animals were only anesthetized but not incised.

Thermal hyperalgesia was assessed by the Hargreaves method (16). Noxious heat sensitivity was measured using a radiant heat device (Ugo Basile, Italy) directed at the plantar surface of the hind paw. A timer was automatically activated with the light source and response latency was defined as the time (s) required for the paw to show an abrupt withdrawal from the increasing temperature. Thermal exposure was interrupted after $20 \mathrm{~s}$ (cut off) to avoid tissue damage. Measurements were made three times with an interval of at least 5 min between tests. Paw withdrawal thresholds in response to mechanical stimuli were assessed using the electronic von Frey method (17). Briefly, rats were placed in individual Plexiglas chambers with a plastic mesh floor and allowed to acclimate to the apparatus for at least 15 min before beginning the test. The tactile threshold was measured using an electronic von Frey anesthesiometer (Insight, Brazil), which was applied vertically and directly to the incision. The force $(\mathrm{g})$ needed to produce a paw withdrawal response was tested at 10-s intervals, 6 times or until 3 similar measurements were obtained. The pain behavior was quantified as the mean of 3 measurements. The individuals conducting the behavioral assays were blind to the treatments.

Data are reported as means \pm SEM and were analyzed by two-way analysis of variance (ANOVA) for repeated measurements with treatment and time as the main factors. Post hoc analyses were performed by the Student-NewmanKeuls test. The level of significance was set at $P<0.05$.

\section{Results}

The incision in the plantar surface of the hind paw produced a significant reduction in thermally stimulated paw withdrawal latencies of the injured limb at all postoperative time points studied (Figure 1). Two-way repeated measures ANOVA applied to paw withdrawal latencies in response 

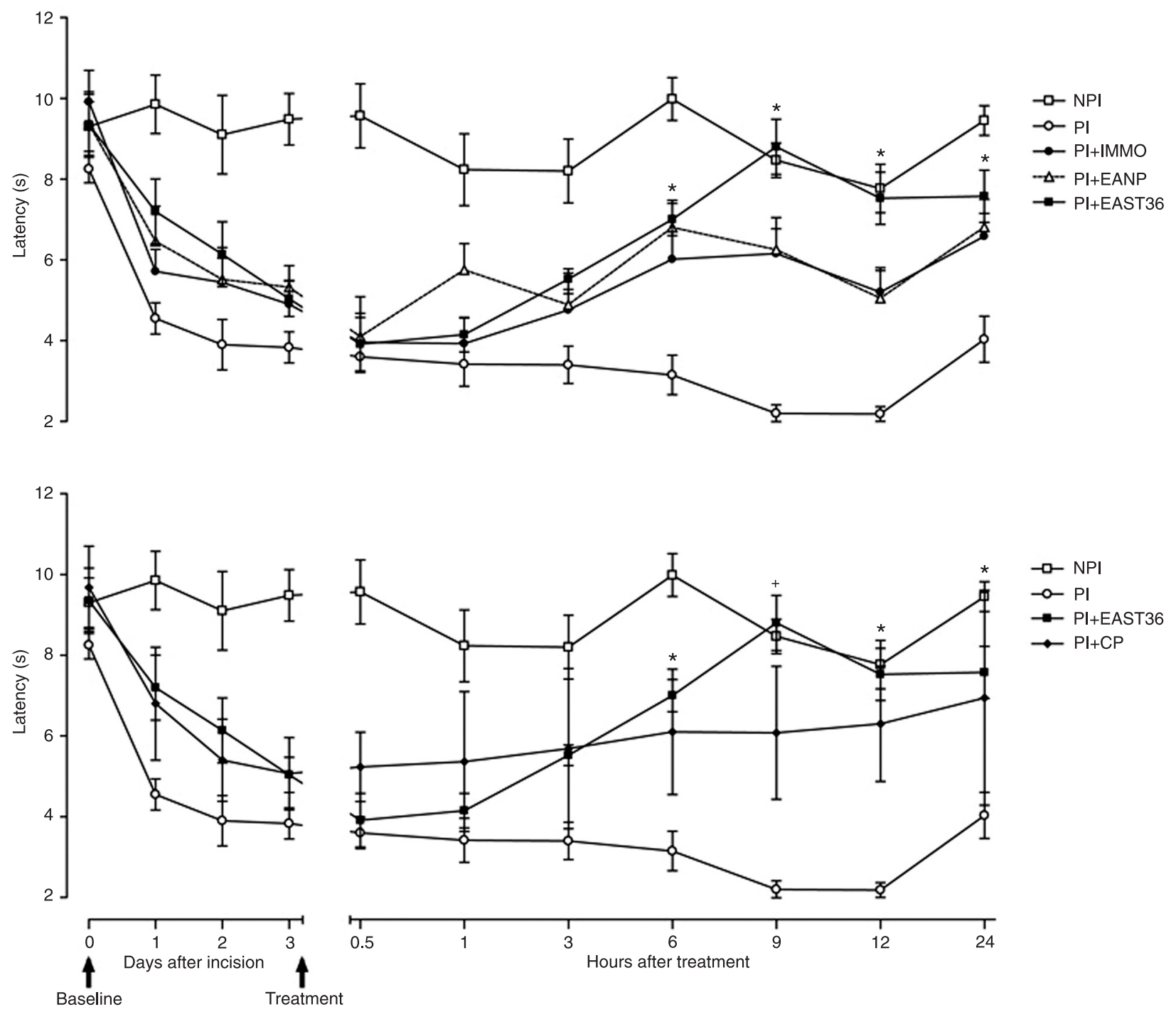

Figure 1. Effect of electroacupuncture and carprofen on thermal hyperalgesia in the plantar incision (PI) model in rats (Hargreaves test). $A$, Comparison of animals incised and subjected to electroacupuncture $(100 \mathrm{~Hz})$ at the Zusanli point $(\mathrm{PI}+\mathrm{EAST} 36 \mathrm{group}, \mathrm{N}=8)$, incised and subjected to electroacupuncture $(100 \mathrm{~Hz})$ at a non-point region (PI+EANP, $N=8)$, incised and subjected to immobilization $\left(\mathrm{PI}+\mathrm{IMMO}, \mathrm{N}=7\right.$ ), only incised ( $\mathrm{PI}, \mathrm{N}=6$ and non-incised animals ( $\mathrm{NPI}$ group, $\mathrm{N}=6$ ). Data are reported as means $\pm \mathrm{SEM}$. ${ }^{*} \mathrm{P}<0.005$ for the PI+EAST36, PI+IMMO, and PI+EANP groups compared to PI (two-way ANOVA for repeated measurements followed by the Student-Newman-Keuls test). B, Comparison between the PI+CP group (animals incised and subjected to carprofen, $2 \mathrm{mg} / \mathrm{kg}, \mathrm{po}, \mathrm{N}$ = 9) and PI+EAST36, PI and NPI groups. ${ }^{*} \mathrm{P}<0.005$ for PI+EAST36 and PI+CP compared to $\mathrm{PI}$; ${ }^{+} \mathrm{P}<0.05$ only for the $\mathrm{PI}+\mathrm{EAST} 36$ group compared to PI (two-way ANOVA for repeated measurements followed by the Student-Newman-Keuls test). PI was different from NPI at all time points after incision.

to thermal stimulation revealed significant effects of treatment $\left(F_{(5,483)}=28.841 ; P<0.001\right)$ and time $\left(F_{(10,483)}=\right.$ $21.054 ; P<0.001)$ and a significant interaction $\left(F_{(50,483)}=\right.$ 1.632; $\mathrm{P}=0.006)$. The post hoc Student-Newman-Keuls test showed that the PI+EAST36, PI+CP, PI+EANP, and $\mathrm{PI}+\mathrm{IMMO}$ groups were similar and had thermally stimulated paw withdrawal latencies that were significantly higher than those of the PI group at 6, 9, 12, and $24 \mathrm{~h}$ after treatment ( $P<0.001$ for all time points), except for the $\mathrm{PI}+\mathrm{CP}$ group that was similar to the PI group at $9 \mathrm{~h}$ (Figure 1).

Similar to thermal hyperalgesia, the plantar incision produced a significant reduction in mechanically stimulated paw withdrawal thresholds in the injured limb at all time points studied (Figure 2). Pairwise multiple comparisons of groups at each time point only detected effects of the treatments $6 \mathrm{~h}$ after their administration. Two-way repeated measures ANOVA applied to paw withdrawal thresholds in response to mechanical stimuli detected significant differ- 

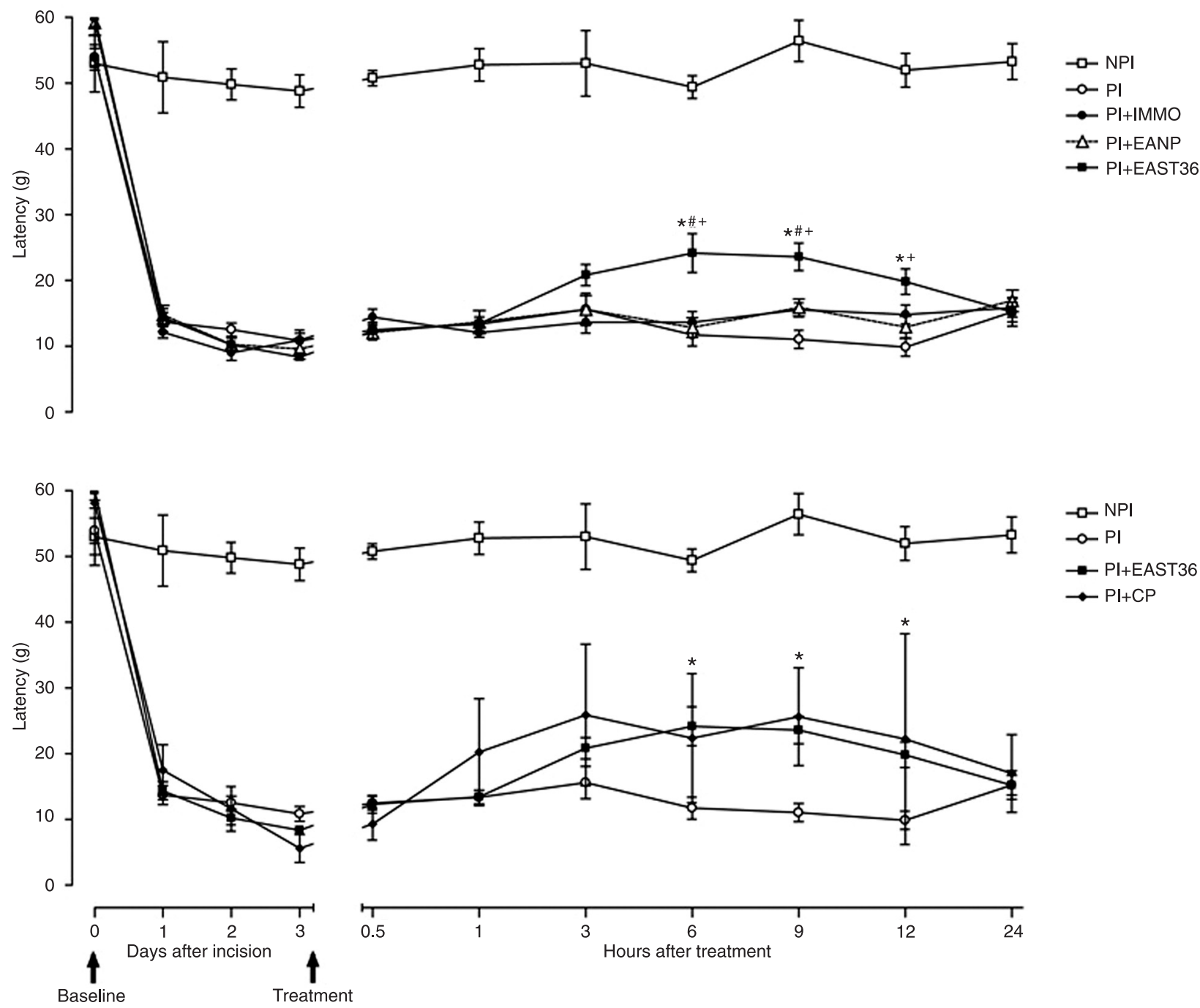

Figure 2. Effect of electroacupuncture and carprofen on mechanical hyperalgesia in the plantar incision (PI) model in rats (von-Frey method). A, Comparison of animals incised and subjected to electroacupuncture $(100 \mathrm{~Hz})$ at the Zusanli point (PI+EAST36 group, N $=8)$, incised and subjected to electroacupuncture $(100 \mathrm{~Hz})$ at a non-acupoint region (PI+EANP, $\mathrm{N}=8)$, incised and subjected to immobilization ( $\mathrm{PI}+\mathrm{IMMO}, \mathrm{N}=7$ ), only incised ( $\mathrm{PI}, \mathrm{N}=6$ and non-incised animals (NPI group, $\mathrm{N}=6$ ). Data are reported as means $\pm \mathrm{SEM}$. ${ }^{*} \mathrm{P}<0.005$ for $\mathrm{PI}+\mathrm{EAST} 36$ compared to $\mathrm{PI} ;{ }^{\#} \mathrm{P}<0.05$ for PI+EAST36 compared to PI+IMMO; ${ }^{+} \mathrm{P}<0.05$ for $\mathrm{PI}+\mathrm{EAST} 36$ compared to $\mathrm{PI}+\mathrm{EANP}$ (two-way ANOVA for repeated measurements followed by the Student-Newman-Keuls test). $B$, Comparison between the $\mathrm{PI}+\mathrm{CP}$ group (animals incised and subjected to carprofen, $2 \mathrm{mg} / \mathrm{kg}, \mathrm{po}, \mathrm{N}=9$ ) and PI+EAST36, PI and NPI groups. ${ }^{*} \mathrm{P}<0.05$ for $\mathrm{PI}+\mathrm{EAST} 36$ and $\mathrm{PI}+\mathrm{CP}$ compared to $\mathrm{PI}$. PI was different from NPI at all time points after incision.

ences due to the treatment $\left(F_{(5,472)}=162.568 ; P<0.001\right)$ and time $\left(F_{(10,472)}=181.261 ; P<0.001\right)$ factors and $a$ significant interaction between them $\left(F_{(50,472)}=7.413\right.$; $P$ $<0.001)$. The PI+EAST36 and PI+CP groups were similar and showed higher paw withdrawal thresholds at 6, 9, and $12 \mathrm{~h}$ after treatment compared to the $\mathrm{PI}, \mathrm{PI}+\mathrm{IMMO}$ and $\mathrm{PI}+\mathrm{EANP}$ groups. There was no difference within the latter groups at any time point.

\section{Discussion}

The present investigation sought to study and com- pare the effect of EA at the Zusanli point (ST36; $100 \mathrm{~Hz}$, $20 \mathrm{~min}$ ) on thermal and mechanical hyperalgesia induced by a rat model of incisional pain to the effect of an NSAID (carprofen) widely used in controlling postoperative pain. Since EA was performed on immobilized animals, we had control groups of animals subjected only to immobilization and animals subjected to EA in a non-acupoint region. Our results showed that EA at the ST36 acupoint produced a significant reduction of mechanical hyperalgesia compared to control groups (PI+IMMO and PI+EANP), and this effect was similar to that obtained with treatment with CP. On the other hand, in the Hargreaves test all treated groups 
(PI+IMMO, PI+EANP, PI+EAST36, and PI+CP) showed a significant reduction in thermal hyperalgesia, with no statistical difference between them.

Taken together, these results indicate that EA at the Zusanli acupoint produced a decline in mechanical hyperalgesia induced by the plantar incision pain model, and this effect was related to stimulation of a specific body region (in this case, the Zusanli point) and not to electrostimulation per se or to immobilization. In contrast, in the Hargreaves test, although the EA at the Zusanli acupoint tended to produce a greater effect, the immobilization and EA in a non-acupoint region also produced a significant reduction of thermal hyperalgesia. In this case, the stress induced by immobilization and electrostimulation was more effective against thermal than mechanical hyperalgesia. It has been shown that drugs can produce a greater effect on thermal than on mechanical hyperalgesia (18).

These results are consistent with those of Oliveira and Prado (11), who reported that $15 \mathrm{~min}$ of EA at $4 \mathrm{~Hz}$ at the Zusanli (ST36) and Sanyinjiao (SP6) acupoints reduced mechanical hyperalgesia in the same pain model. Both studies provide positive results, although there are important differences in the experimental protocols: the frequency of electric stimulation ( $100 \mathrm{~Hz}$ vs $4 \mathrm{~Hz}$ ), combination of points (ST36 vs ST36 + SP6), time of stimulation (20 vs $15 \mathrm{~min}$ ), type of restraint (immobilization vs light halothane anesthesia), and time after surgery when the stimulus was applied (third day vs $2 \mathrm{~h}$ ). Stimulation of 15 vs 20 min does not appear to result in significant differences in the pattern of analgesic response induced by EA (13). Moreover, EA at ST36 or simultaneously at SP6 and ST36 is commonly used to induce analgesia, and until now no study has explored the differences between these combinations of points. However, the different frequencies of stimulation could result in different degrees of anti-inflammatory and analgesic effects due to different neural pathways and neurotransmitters. The analgesia induced by EA at low frequencies $(4 \mathrm{~Hz})$ is mainly mediated by encephalin and $\mu$ and $\delta$ receptors, whereas analgesia induced by EA at high frequencies $(>100 \mathrm{~Hz})$ is mainly mediated by the release of dynorphins and $\mathrm{k}$ receptors (19). Furthermore, different frequencies of stimulation are linked to different patterns of neurotransmitter release, including substance $P$ and cholecystokinin, as well as different brain nuclei; for example, lesions in the parabrachial nucleus can only block the analgesia induced by EA at high frequencies, while lesions in the arcuate nucleus can block the analgesia induced by EA at low frequencies (19). A frequency of $100 \mathrm{~Hz}$ was chosen based on previous data from our laboratory (14).

The type of restraint used to perform EA is another important issue in the experimental protocols of acupuncture studies. In the present study, EA was performed in conscious animals subjected to immobilization. Despite the disadvantage of the effect of immobilization stress on analgesic responses and on the inflammatory process, this method has been used in acupuncture research to avoid the influence of anesthesia (14). Even considering the respective differences and limitations of immobilization and anesthetic procedures, if the appropriate controls are used, both methods are considered acceptable.

Another important aspect of the PI model is the time after surgery when the treatment is performed. Clinical studies on the effect of acupuncture on postoperative pain have performed acupuncture at several different times: only before, only after, before, and after surgery and even with continuous treatment for 3-6 days after surgery (see Ref. 9). In the present study, all treatments were applied on the third day after surgery when the mechanism of hypersensitivity is well established, and mechanical and thermal hyperalgesia is maximal (5). Oliveira and Prado (11) studied the effect of EA in the early stages of hyperalgesia ( $2 \mathrm{~h}$ after the surgery) when sensitization mechanisms are being generated (4). Due to the positive results of both studies (with EA performed either $2 \mathrm{~h}$ or on the 3rd day after surgery), we can speculate that EA at acupoints possibly reduces the mechanisms responsible for the generation and maintenance of induced hyperalgesia in the incisional pain model.

Our results also showed that EA at the ST36 acupoint produced a reduction in thermal hyperalgesia similar to $\mathrm{CP}$ at several of the time points studied. Recently, the effects of acupuncture have been compared to those of NSAIDs: Lau et al. (20) have demonstrated that, relative to the COX-2 inhibitor celecoxib, EA applied to the acupoints Zusanli (ST36) and Sanyinjiao (SP6) (2 Hz, 0.5-1-2 mA) has a longer-lasting and greater analgesic effect in reducing neuropathic hypersensitivity induced by the spinal nerve ligation model. Although this effect of acupuncture on behavioral tests was followed by a reduced expression of COX-2 in the spinal L4-L6 dorsal horn, the authors admit that the mechanism of acupuncture analgesia is beyond the inhibition of COX-2 (20).

In summary, we showed that EA applied at $100 \mathrm{~Hz}$ at the ST36 point, but not at non-acupoints, can reduce mechanical pain in the rat model of incisional pain, and its effectiveness is comparable to that of CP. Therefore, EA may represent an additional intervention for the treatment of postoperative incisional pain.

\section{Acknowledgments}

Research supported by CAPES. F.M. Teixeira, L.L. Castro, R.T. Ferreira, and P.A. Pires were recipients of CAPES fellowships. 


\section{References}

1. Kehlet $\mathrm{H}$, Holte K. Effect of postoperative analgesia on surgical outcome. Br J Anaesth 2001; 87: 62-72.

2. Pavlin DJ, Chen C, Penaloza DA, Polissar NL, Buckley FP. Pain as a factor complicating recovery and discharge after ambulatory surgery. Anesth Analg 2002; 95: 627-634.

3. Hunt SP, Mantyh PW. The molecular dynamics of pain control. Nat Rev Neurosci 2001; 2: 83-91.

4. Brennan TJ, Vandermeulen EP, Gebhart GF. Characterization of a rat model of incisional pain. Pain 1996; 64: 493501.

5. Whiteside GT, Harrison J, Boulet J, Mark L, Pearson M, Gottshall S, et al. Pharmacological characterisation of a rat model of incisional pain. Br J Pharmacol 2004; 141: 85-91.

6. Roughan JV, Flecknell PA. Behaviour-based assessment of the duration of laparotomy-induced abdominal pain and the analgesic effects of carprofen and buprenorphine in rats. Behav Pharmacol 2004; 15: 461-472.

7. MacPhail CM, Lappin MR, Meyer DJ, Smith SG, Webster $\mathrm{CR}$, Armstrong PJ. Hepatocellular toxicosis associated with administration of carprofen in 21 dogs. J Am Vet Med Assoc 1998; 212: 1895-1901.

8. Cheng SB, Ding LK. Practical application of acupuncture analgesia. Nature 1973; 242: 559-560.

9. Sun Y, Gan TJ, Dubose JW, Habib AS. Acupuncture and related techniques for postoperative pain: a systematic review of randomized controlled trials. $\mathrm{Br} J$ Anaesth 2008; 101: 151-160.

10. Song MJ, Wang YQ, Wu GC. Additive anti-hyperalgesia of electroacupuncture and intrathecal antisense oligodeoxynucleotide to interleukin-1 receptor type I on carrageenaninduced inflammatory pain in rats. Brain Res Bull 2009; 78: 335-341.

11. Oliveira R, Prado WA. Anti-hyperalgesic effect of electroacupuncture in a model of post-incisional pain in rats. Braz
J Med Biol Res 2000; 33: 957-960.

12. Zimmermann M. Ethical guidelines for investigations of experimental pain in conscious animals. Pain 1983; 16: 109-110.

13. Romita VV, Suk A, Henry JL. Parametric studies on electroacupuncture-like stimulation in a rat model: effects of intensity, frequency, and duration of stimulation on evoked antinociception. Brain Res Bull 1997; 42: 289-296.

14. Medeiros MA, Canteras NS, Suchecki D, Mello LE. c-Fos expression induced by electroacupuncture at the Zusanli point in rats submitted to repeated immobilization. Braz $J$ Med Biol Res 2003; 36: 1673-1684.

15. Camprodon RA, Bowles MJ. Perioperative analgesia in experimental small bowel transplantation. Transplant Proc 2006; 38: 1857-1858.

16. Hargreaves K, Dubner R, Brown F, Flores C, Joris J. A new and sensitive method for measuring thermal nociception in cutaneous hyperalgesia. Pain 1988; 32: 77-88.

17. Moller KA, Johansson B, Berge OG. Assessing mechanical allodynia in the rat paw with a new electronic algometer. $J$ Neurosci Methods 1998; 84: 41-47.

18. Campillo A, Gonzalez-Cuello A, Cabanero D, GarciaNogales P, Romero A, Milanes MV, et al. Increased spinal dynorphin levels and phospho-extracellular signal-regulated kinases 1 and 2 and c-Fos immunoreactivity after surgery under remifentanil anesthesia in mice. Mol Pharmacol 2010; 77: 185-194

19. Han JS. Acupuncture: neuropeptide release produced by electrical stimulation of different frequencies. Trends $\mathrm{Neu}$ rosci 2003; 26: 17-22.

20. Lau WK, Lau YM, Zhang HQ, Wong SC, Bian ZX. Electroacupuncture versus celecoxib for neuropathic pain in rat SNL model. Neuroscience 2010; 170: 655-661. 